\title{
Measurement of $\beta$-hydroxybutyrate in capillary blood obtained from an ear to detect hyperketonemia in dairy cows by using an electronic handheld device
}

\author{
D. Süss, ${ }^{*}$ M. Drillich, ${ }^{*}$ D. Klein-Jöbstl, ${ }^{*}$ K. Wagener, ${ }^{*}$ S. Krieger, ${ }^{*}$ A. Thiel, ${ }^{*}$ L. Meyer,† I. Schwendenwein,† \\ and $M$. Iwersen ${ }^{* 1}$ \\ ${ }^{*}$ Clinical Unit for Herd Health Management in Ruminants, University Clinic for Ruminants, Department for Farm Animals \\ and Veterinary Public Health, University of Veterinary Medicine Vienna, 1210 Vienna, Austria \\ †FirstFarms Slovakia, 90068 Plavecký Štvrtok, Slovakia \\ †Central Clinical Pathology Unit, Department for Pathobiology, University of Veterinary Medicine Vienna, 1210 Vienna, Austria
}

\begin{abstract}
The primary objective of the present study was to test whether capillary blood obtained by puncturing the skin of an ear with a minimal invasive lancet technique is able to detect hyperketonemia (HYK) in dairy cows. Furthermore, test characteristics of a new available handheld device, the FreeStyle Precision Neo (FSP-Neo, Abbott GmbH \& Co. KG, Wiesbaden, Germany) for determination of $\beta$-hydroxybutyrate (BHB) concentrations in bovine blood were evaluated by comparing the measurements with a laboratory reference. The BHB concentration was determined with the FSP-Neo device in 720 capillary blood samples from 3 different sampling sites (left, right ear, and repeated measurement) and in 240 samples from a coccygeal vessel. The concentration of BHB in serum harvested from the coccygeal blood samples was analyzed at the laboratory and was used as reference. The Spearman correlation coefficient $\left(\rho_{\mathrm{s}}\right)$ between the BHB concentrations in capillary blood measured with the handheld device and the reference test was between 0.76 and 0.81 . Using capillary blood, the mean \pm standard deviation BHB difference compared with the reference test was $0.20 \pm 0.47 \mathrm{mmol} / \mathrm{L}$ for all 3 sampling locations at the ears. The receiver operating characteristic analyses for the FSP-Neo device resulted in an optimized threshold for the detection of subclinical ketosis (SCK) in capillary blood of $1.3 \mathrm{mmol} / \mathrm{L}$ (left and right ear) and $1.2 \mathrm{mmol} / \mathrm{L}$ (repeated measurements). Applying these adjusted threshold sensitivities (Se) for all 3 capillary sampling sites at the ear were $100 \%$, and specificities (Sp) ranged between 93 and 94\%. Hence, we conclude that all sampling locations were suitable to identify
\end{abstract}

Received January 18, 2016.

Accepted May 4, 2016.

${ }^{1}$ Corresponding author: Michael.Iwersen@vetmeduni.ac.at cows suffering from SCK. The reference test compared with BHB measurements in coccygeal blood resulted in a $\rho_{\mathrm{s}}$ of 0.92 with a mean \pm standard deviation of 0.02 $\pm 0.21 \mathrm{mmol} / \mathrm{L}$. The receiver operating characteristic analyses for the FSP-Neo device resulted in an optimized threshold for the detection of SCK in coccygeal blood of $1.1 \mathrm{mmol} / \mathrm{L}$, with a corresponding Se and Sp of 100 and $95 \%$, respectively. Because capillary blood is easily achievable from an ear, particularly if animals are fixed in headlocks for routine checkups, this technique is considered as an additional minimally invasive method for the identification of dairy cows suffering from HYK.

Key words: dairy cow, hyperketonemia, $\beta$-hydroxybutyrate, capillary blood

\section{INTRODUCTION}

Ketosis in its subclinical (SCK) and clinical manifestation is a widespread metabolic disease in high-producing dairy cows and occurs predominantly in the first weeks postpartum. Analyzing the BHB concentration in serum or plasma is the reference test for detecting hyperketonemia (HYK), comprising clinical ketosis and SCK (Duffield et al., 1998). According to Oetzel (2004), clinical ketosis is defined as BHB concentrations in blood $\geq 3.0 \mathrm{mmol} / \mathrm{L}$, and $\mathrm{SCK}$ is defined as a BHB concentration of 1.2 to $2.9 \mathrm{mmol} / \mathrm{L}$ in blood. The average prevalence of SCK in 10 European countries within 2 to $15 \mathrm{~d}$ postpartum (dpp) was $21.8 \%$, using a threshold of $\geq 1.2 \mathrm{mmol}$ of $\mathrm{BHB} / \mathrm{L}$ in whole blood (Suthar et al., 2013). For Western Europe, Berge and Vertenten (2014) presented a prevalence of HYK of $39 \%$ between $\mathrm{d} 7$ and 21 after calving based on a milk test for ketones. Mahrt et al. (2015) reported a mean prevalence of SCK within the first $42 \mathrm{dpp}$ in Germany of $11.8 \%$, using also a threshold of $\geq 1.2 \mathrm{mmol}$ of $\mathrm{BHB} / \mathrm{L}$. Blood from a tail vessel was tested with a handheld device in this study. 
Various electronic handheld devices have been evaluated for the measurement of BHB in the recent years, and all tested devices were eligible as a cow-side test for the detection of SCK (Iwersen et al., 2013; Mahrt et al., 2014; Pineda and Cardoso, 2015). Kanz et al. (2015) tested the suitability of capillary blood obtained from the skin of the external vulva for BHB measurements using different handheld devices in dairy cows. The authors reported that this technique is eligible to detect SCK. Obtaining ear capillary blood can be considered as an alternate for cow-side determination of BHB in situations in which sampling from other vessels is difficult or not possible.

To our knowledge, the determination of BHB concentration in capillary blood obtained from an ear using a minimally invasive sampling technique has not been tested yet in cattle.

The primary objective of the present study was to test whether capillary blood obtained by puncturing the skin of an ear with a minimally invasive lancet is eligible as an additional technique to detect HYK in dairy cows. Furthermore, test characteristics of a new available handheld device for determination of the BHB concentrations in bovine blood were evaluated by comparing the measurements with a laboratory reference.

\section{MATERIALS AND METHODS}

The study was approved by the institutional ethics committee of the University of Veterinary Medicine (ETK-10/05/2015), Vienna, as well as by the Slovakian Regional Veterinary Food Administration.

The study was conducted in August 2015 on a commercial dairy farm in Slovakia, housing approximately 2,500 Holstein-Friesian cows. Animals were kept on deep straw bedding in a freestall barn. The average ECM yield (based on $4.0 \%$ butterfat and $3.4 \%$ protein) was $9,165 \mathrm{~kg}$ in 2014. To achieve a maximum irrelevant difference in the BHB concentration of $0.1 \mathrm{mmol} / \mathrm{L}$ between the laboratory and capillary blood results with the handheld device, a sample size calculation (type I error $\alpha=0.05$, type II error $\beta=0.2$ ) was performed, resulting in 216 animals required. To compensate for possible data loss due to study animal exclusions [e.g., because of (pre-)analytical problems], 240 animals were sampled and used in this study. The enrollment of study animals was based on the risk period for the occurrence of HYK reported by Oetzel (2004). Hence, all multiparous cows between 1 to 21 dpp were tested once for HYK during the study period.

An electronic handheld device [FreeStyle Precision Neo (FSP-Neo), Abbott GmbH \& Co. KG, Wiesbaden, Germany)] was used to analyze the BHB concentration in capillary and coccygeal blood. To gain capillary blood, a disposable safety lancet (MiniCollect Safety Lancet, Greiner Bio-One International AG, Kremsmünster, Austria) was used, with a penetration depth of $2 \mathrm{~mm}$ and a blade width of $1.5 \mathrm{~mm}$. For sampling procedures, the cows were fixed in headlocks and only restrained manually for collecting a single drop of capillary blood at any position of the ears edge. Because no or only minor defensive reactions of the cows were observed, no further restraints were necessary.

Capillary blood samples were taken at 3 different locations: at the edge of the left and right ear and again on one of the 2 ears. The repeated measurement (2nd test) was performed alternately on one of the 2 ears approximately $1 \mathrm{~cm}$ lateral from the first puncture and regarded as a third sampling location for capillary blood (i.e., to evaluate a possible influence of the sampling location within one ear). The sampling procedures started with cleaning and puncturing the skin of the left or right ear. The amount of blood necessary for BHB measurements with the FSP-Neo was $1.5 \mu \mathrm{L}$. If the blood volume was insufficient for the measurement, the capillary bleeding was enforced by softly squeezing the skin of the ear. If the obtained blood volume was still insufficient for a reliable measurement, the ear was punctured once again. After inserting the test strip into the handheld device, the front edge of the strip was dipped directly onto the drop of blood. Additionally, blood samples from a coccygeal vessel from each cow were obtained with vacuum tubes coated with a clot activator for serum collection (Vacuette, $9 \mathrm{~mL}$, Greiner Bio-One GmbH, Kremsmünster, Austria). Immediately after sampling, the BHB concentration in whole blood was determined by dipping the sensor of the test strip from the FSP-Neo device onto the surface of the bloodfilled serum tube. The coccygeal blood samples were analyzed with the FSP-Neo device to compare the test results for capillary and coccygeal blood and to describe test characteristics of the handheld meter. Therefore, the BHB concentration of the coccygeal blood sample was determined in serum at the laboratory and used as reference.

After clotting at approximately $15^{\circ} \mathrm{C}$ for $2 \mathrm{~h}$, the reference sample was centrifuged $\left(10 \mathrm{~min}, 18^{\circ} \mathrm{C}, 2,200 \times g\right.$; Eppendorf Centrifuge 5804, Eppendorf AG, Hamburg, Germany) to harvest the serum. The supernatant was divided in 2 aliquots and stored at a temperature of $-18^{\circ} \mathrm{C}$ until further analyses at the laboratory of the Central Clinical Pathology Unit (CCPU), University of Veterinary Medicine, Vienna, Austria. Laboratory proceedings for the determination of the BHB concentration at the CCPU were previously described by Pichler et al. (2014).

Furthermore, 20 aliquot blood samples taken from the same cow were randomly placed between the samples 
obtained from the 240 study animals. These standard samples were taken to evaluate the intra-assay variability of the laboratory analyses. Additionally, the intraand interassay coefficients of variation were calculated for the FSP-Neo device in capillary blood. To evaluate the variability of the handheld device measurements in capillary blood, 3 cows sampled with laboratory BHB concentrations varying between 0.36 and $1.86 \mathrm{mmol} / \mathrm{L}$ were used. Each animal was tested 5 times with the same device (intra-assay variability) and 5 times with different devices of the same type (inter-assay variability). The date of sampling, the animals identification number, the number of required punctures, squeezing, and the measured BHB concentrations of the handheld device in capillary and coccygeal blood were recorded onto a pre-assigned data sheet.

\section{Statistical Analyses}

SPSS Statistics for Windows (version 20.0; IBM Deutschland GmbH, Ehningen, Germany), Excel for Windows (version 14.0, Microsoft Corp., Redmond, WA), BiAS for Windows (version 10.06; Epsilon-Verlag, Darmstadt, Germany), and MedCalc for Windows (version 12.4; MedCalc Software, Ostend Belgium) were used for statistical analyses. For all statistical tests, the level of significance was set at $P<0.05$.

Data were tested for normal distribution by the Kolmogorov-Smirnov test and based on this nonparametric testing was performed. To describe the statistical association between variables, Spearman's correlation coefficients $\left(\boldsymbol{\rho}_{\mathrm{s}}\right)$ were calculated for the BHB concentrations analyzed with the handheld device thrice in capillary blood of the ear (i.e., left, right, repeated measurement) and in coccygeal blood, and with the reference method in the laboratory. The agreement between BHB concentrations determined with the handheld device and the values determined at the CCPU was evaluated by using the method described by Bland and Altman (1986).

According to the concentration of BHB determined in serum at the CCPU, samples were classified as nonketotic $(<1.20 \mathrm{mmol} / \mathrm{L})$ or (subclinically) ketotic $(\geq 1.20 \mathrm{mmol} / \mathrm{L})$. Based on this classification, receiver operating characteristics (ROC) analyses were performed to calculate optimal thresholds for all 4 sampling locations (i.e., left, right ear, repeated measurement, coccygeal vessel). Sensitivities (Se), specificities $(\mathbf{S p})$ and the Youden index (YI) for these optimized thresholds were reported. The YI was calculated as $(\mathrm{Se}+\mathrm{Sp}-1)$, representing the number of all correctly identified outcomes (Youden, 1950). The area under the ROC curve (AUROC) shows how well an estimated threshold can differentiate between 2 diagnostic groups (i.e., nonketotic vs. subclinically ketotic) and represents the quality of this parameter according to Se and Sp (Swets, 1988). The AUROC is equivalent to the probability that a randomly chosen positive instance (e.g., a cow tested positive for SCK) has a higher test result than a randomly chosen negative instance (e.g., a cow tested negative for SCK; Fawcett, 2006).

Moreover, mixed model analyses for repeated measurements were performed to evaluate if the sampling location and squeezing (classified in 2 different groups, i.e., the amount of blood was sufficient: a nonsqueezing group, and the amount was insufficient for valid testing: a squeezing group) affected the BHB concentration.

\section{RESULTS}

In total, samples were taken from 240 dairy cows that were on average in the third lactation $[$ minimum $=2$, maximum $=7$, interquartile range $(\mathrm{IQR})=1$ ] with a median of $7 \mathrm{dpp}$ (minimum $=0$, maximum $=21$, IQR $=8$ ). A total number of 25 cows exceeded a threshold of $1.2 \mathrm{mmol} / \mathrm{L}$ of BHB in serum, resulting in a prevalence of SCK on farm of $10.4 \%$. The median and mean \pm standard deviation BHB concentration determined at the CCPU from all samples was $0.63 \mathrm{mmol} / \mathrm{L}$ (minimum $=0.3 \mathrm{mmol} / \mathrm{L}$, maximum $=4.7 \mathrm{mmol} / \mathrm{L}, \mathrm{IQR}$ $=0.2 \mathrm{mmol} / \mathrm{L}$ ) and $0.76 \pm 0.57 \mathrm{mmol} / \mathrm{L}$, respectively. Laboratory results and further descriptive statistical parameters for the BHB concentrations measured in capillary and coccygeal blood using the FSP-Neo device are presented in Table 1.

Capillary blood from the ear could be obtained in $88.5 \%$ with the first puncture and in $98.8 \%$ with the first or second puncture. Altogether, 720 capillary blood samples and 240 samples from the coccygeal vessel were tested with the FSP-Neo device.

The comparison of BHB concentrations measured with the FSP-Neo at 3 different capillary blood sampling locations with the reference resulted in a $\rho_{\mathrm{s}}$ of $0.78,0.76$, and 0.81 for the left ear, the right ear, and the repeated measurement, respectively (Figure 1, $P$ $<0.01$, for each parameter). Comparing the results of capillary blood from the different sampling sites yielded in $\rho_{\mathrm{s}}=0.77$ between the left and right ear and in $\rho_{\mathrm{s}}=$ 0.81 between the repeated measurement and the left and right ear, respectively $(P<0.01)$. The $\rho_{\mathrm{s}}$ for the BHB concentration in the coccygeal blood measured with the handheld device and the reference test was $0.92(P<0.01)$.

The Bland and Altman plots in Figures 2a and 2b depict the differences between the BHB concentrations measured with the handheld device and the reference test. Using capillary blood, the mean \pm standard deviation BHB deviation against the reference test were 0.20 $\pm 0.47 \mathrm{mmol} / \mathrm{L}$ for all 3 capillary sampling locations, 
Table 1. Descriptive statistics of the BHB concentrations measured in capillary and coccygeal blood using the handheld device as well as in serum analyzed at the laboratory

\begin{tabular}{|c|c|c|c|c|c|}
\hline \multirow{2}{*}{ Parameter } & \multirow{2}{*}{$\frac{\text { Serum }^{2}}{\text { Laboratory } \mathrm{CCPU}^{3}}$} & \multicolumn{4}{|c|}{ FSP-Neo $^{1}$} \\
\hline & & $\frac{\text { Coccygeal blood }}{\text { Tail vessel }}$ & \multicolumn{3}{|c|}{ Capillary blood } \\
\hline Number of samples & 240 & 240 & 240 & 240 & 240 \\
\hline Mean $(\mathrm{mmol} / \mathrm{L})$ & 0.76 & 0.79 & 0.98 & 0.99 & 0.93 \\
\hline $\mathrm{SD}(\mathrm{mmol} / \mathrm{L})$ & 0.57 & 0.66 & 0.73 & 0.72 & 0.71 \\
\hline
\end{tabular}

${ }^{1}$ FreeStyle Precision Neo (Abbott GmbH \& Co. KG, Wiesbaden, Germany).

${ }^{2}$ Obtained from coccygeal blood (reference test).

${ }^{3}$ Laboratory of the Central Clinical Pathology Unit (CCPU), University of Veterinary Medicine, Vienna, Austria.

${ }^{4}$ Second puncture at the ear, approximately $1 \mathrm{~cm}$ lateral from the first one at the left or right ear.

$0.22 \pm 0.49$ for the left ear, $0.23 \pm 0.51$ for the right ear, and $0.17 \pm 0.41 \mathrm{mmol} / \mathrm{L}$ for the second test on the ear (positive biases, $P<0.01$ ). Using coccygeal blood, the mean \pm standard deviation BHB difference compared with the reference test was $0.02 \pm 0.21 \mathrm{mmol} / \mathrm{L}$ (positive bias, $P<0.01$ ).
The mixed model analysis was performed to evaluate whether the sampling site at the ear and squeezing the skin of the ear influenced the difference in BHB concentration between test results obtained from capillary blood at the ear and coccygeal serum analyzed at the CCPU (Table 2). The difference of mean between the

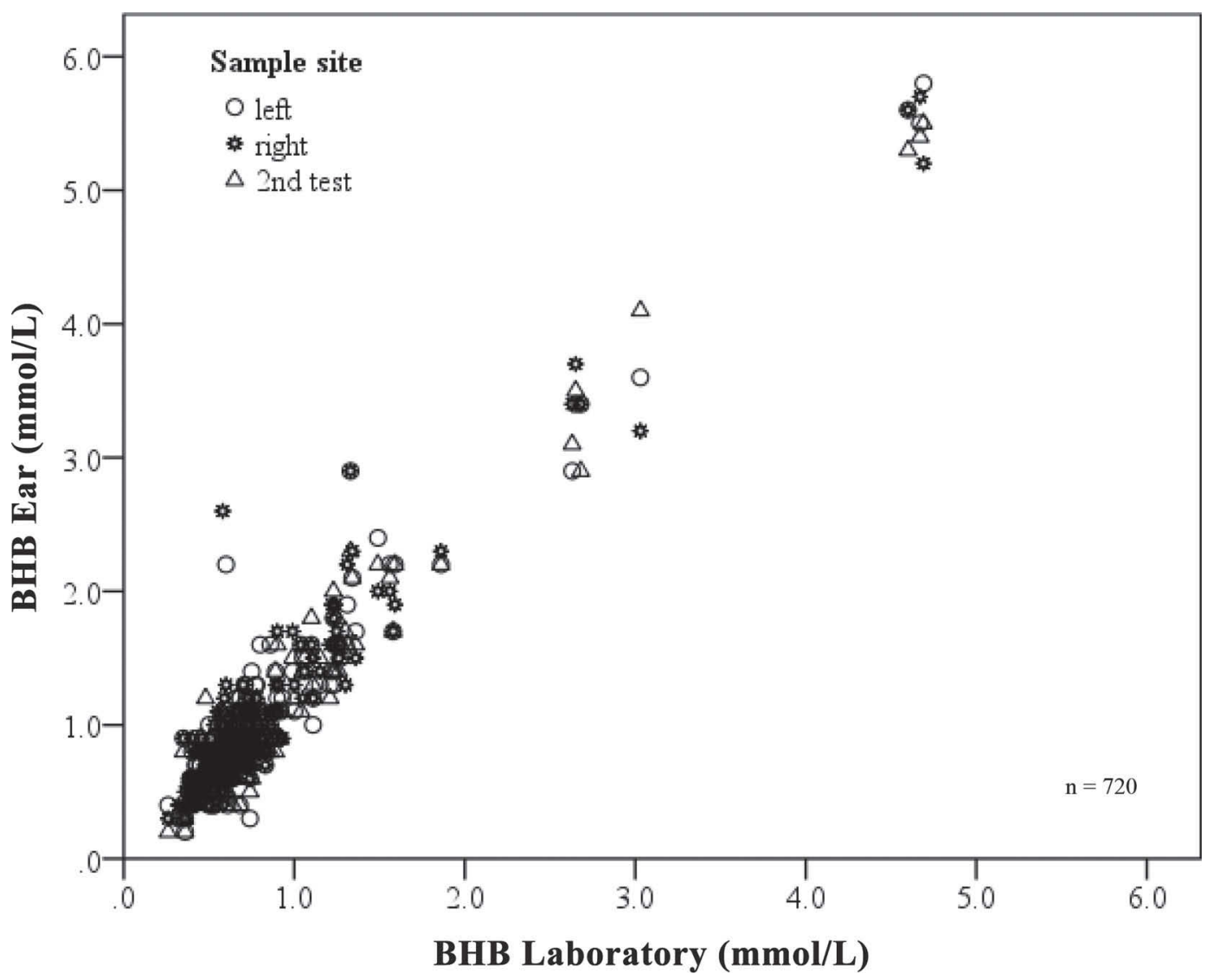

Figure 1. $\beta$-Hydroxybutyrate concentrations $(\mathrm{mmol} / \mathrm{L})$ from the reference test (laboratory) against values obtained with the handheld device at the 3 different capillary sampling sites at the ears. 
A

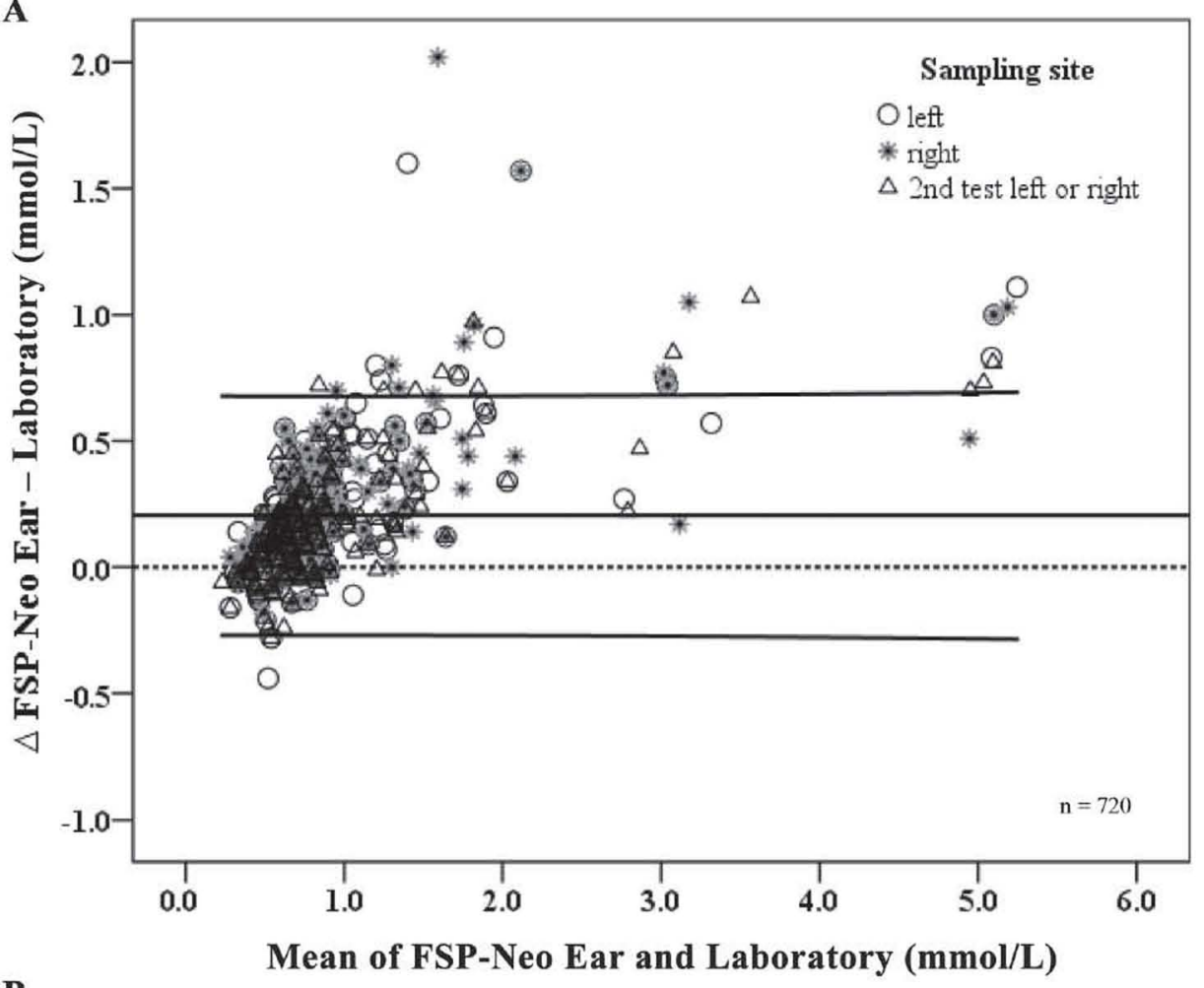

B

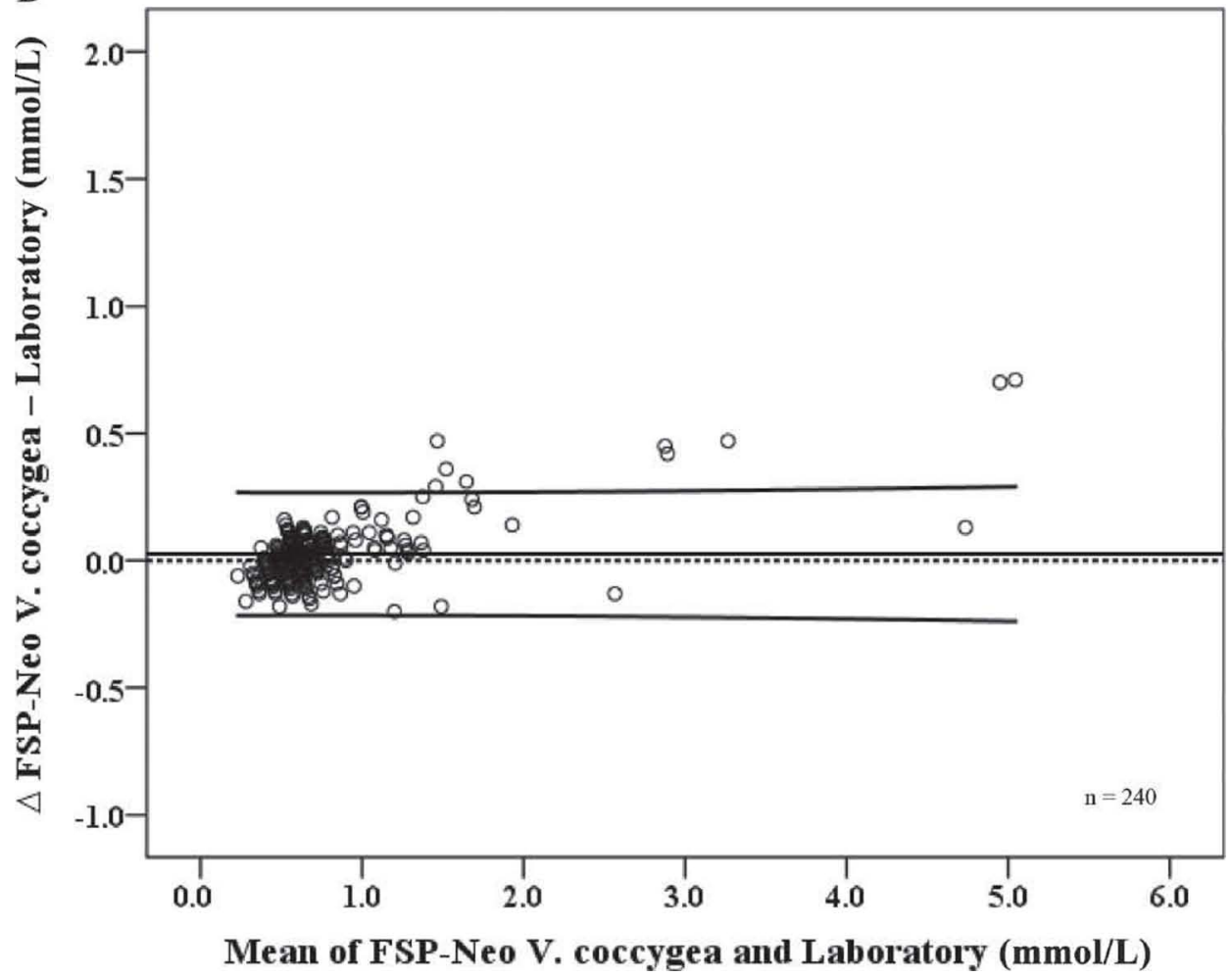

Figure 2. (a) Bland-Altman plot of differences between BHB concentrations obtained at the laboratory in serum and measured in capillary blood at 3 different capillary sampling locations with the FreeStyle Precision Neo (FSP-Neo, Abbott GmbH \& Co. KG, Wiesbaden, Germany). The upper and lower solid lines illustrate the mean $\pm 2 \mathrm{SD}$, and the solid line in the middle shows the mean. The dotted line indicates zero (b) Bland-Altman Plot of differences between BHB concentrations obtained at the laboratory in serum and measured in coccygeal blood with the FSP-Neo. The upper and lower solid lines illustrate the mean $\pm 2 \mathrm{SD}$, and the solid line in the middle shows the mean. The dotted line indicates zero. 
Table 2. Mixed model analyses to show the influence of squeezing and sampling site on the difference in BHB concentrations between test results from capillary blood from the ear and coccygeal serum analyzed at the Central Clinical Pathology Unit (CCPU), University of Veterinary Medicine, Vienna, Austria

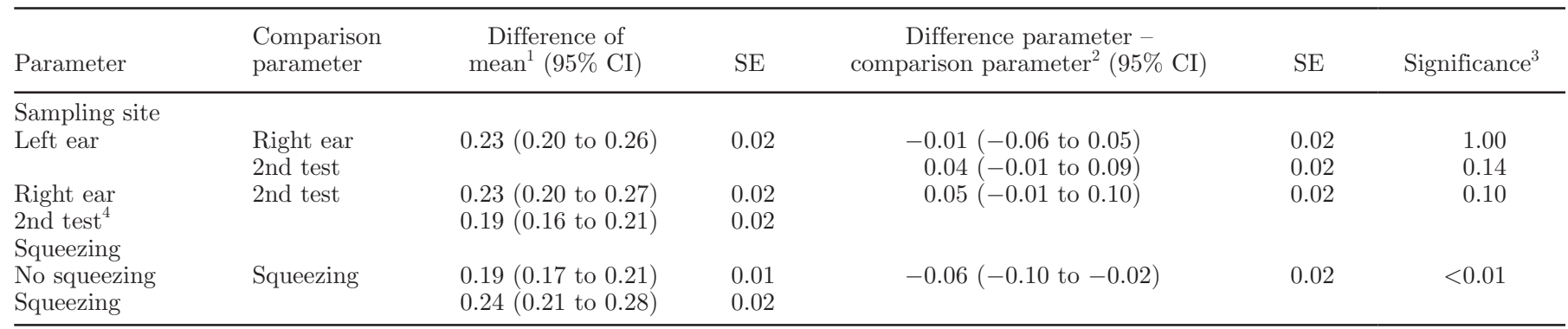

${ }^{1}$ Difference of mean $(\mathrm{mmol} / \mathrm{L})$ of BHB between sampling location and test results determined at the CCPU.

${ }^{2}$ Difference of mean $(\mathrm{mmol} / \mathrm{L})$ of BHB from the parameter (against the reference test) against the difference in mean (mmol/L) from the comparison parameter (against the reference test).

${ }^{3}$ Adjustment for multiple comparisons by Bonferroni.

${ }^{4} 2$ nd test: second puncture at the ear, approximately $1 \mathrm{~cm}$ lateral from the first one at the left or right ear.

sampling locations and the test results determined at the laboratory was $0.19 \mathrm{mmol} / \mathrm{L}$ for the no squeezing group and $0.24 \mathrm{mmol} / \mathrm{L}$ for the squeezing group. This resulted in a significant difference in BHB between the 2 groups (no squeezing/squeezing) of $0.06 \mathrm{mmol} / \mathrm{L}$, which was considered as the effect of squeezing.

Sampling site did not affect the difference between BHB concentrations analyzed with the handheld device in capillary blood and the reference after significancecorrection by Bonferroni (Bland and Altman, 1995). However, significant differences in BHB concentrations in capillary blood compared with the reference were found.

Therefore, ROC analyses were performed to determine optimized thresholds for detecting SCK using the FSP-Neo. A threshold of $1.2 \mathrm{mmol} / \mathrm{L} \mathrm{BHB}$ in serum determined at the CCPU was set to distinguish between animals with SCK and healthy animals. The optimized threshold for using capillary blood was $1.3 \mathrm{mmol} / \mathrm{L}$ of $\mathrm{BHB}$ for the left and right ear and $1.2 \mathrm{mmol} / \mathrm{L}$ of BHB for a repeated testing. Sensitivities for all 3 sampling sites at the ear were $100 \%$. Specificities ranged between 93\% (left ear) and 94\% (right ear, repeated measurement). For the sampling site at the left ear, the YI for capillary blood was lowest with $93 \%$, on the other 2 locations the YI was slightly higher. For all 3 sampling locations for capillary blood (left, right ear, and repeated measurement), the AUROC was $99 \%$ with a corresponding Se of $99 \%$, Sp of $94 \%$, and YI of 93\%. The optimized threshold for the FSP-Neo using coccygeal blood was $1.1 \mathrm{mmol} / \mathrm{L}$ of BHB. The AUROC for coccygeal blood was 100\%, with Se and Sp of 100 and $95 \%$, respectively, and YI of 95\%. Additional test characteristics are presented in Table 3. A pairwise

Table 3. Performances and optimized thresholds of the FSP-Neo to detect SCK in capillary and coccygeal blood based on a threshold for BHB concentration in serum of $1.2 \mathrm{mmol} / \mathrm{L}$

\begin{tabular}{|c|c|c|c|c|c|}
\hline Parameter $^{1}$ & \multicolumn{5}{|c|}{ FSP-Neo ${ }^{2}$} \\
\hline Optimized threshold ${ }^{5}(\mathrm{mmol} / \mathrm{L})$ & 1.3 & 1.3 & 1.2 & 1.3 & 1.1 \\
\hline AUROC $[\%(95 \% \text { CI })]^{\prime}$ & $\begin{array}{l}99 \\
(98-100)\end{array}$ & $\begin{array}{l}99 \\
(98-100)\end{array}$ & $\begin{array}{l}99 \\
(98-100)\end{array}$ & $\begin{array}{l}99 \\
(99-100)\end{array}$ & $\begin{array}{l}100 \\
(99-100)\end{array}$ \\
\hline Sp $[\%(95 \%$ CI $)]$ & $\begin{array}{l}93 \\
(89-96)\end{array}$ & $\begin{array}{l}94 \\
(90-97)\end{array}$ & $\begin{array}{l}94 \\
(89-96)\end{array}$ & $\begin{array}{l}94 \\
(92-96)\end{array}$ & $\begin{array}{l}95 \\
(91-97)\end{array}$ \\
\hline YI $(\%)$ & 93 & 94 & 94 & 93 & 95 \\
\hline
\end{tabular}

${ }^{1}$ AUROC = area under the receiver operating characteristics curve; Se = sensitivity; $\mathrm{Sp}=$ specificity; YI = Youden index.

${ }^{2}$ FreeStyle Precision Neo (Abbott GmbH \& Co. KG, Wiesbaden, Germany).

${ }^{3}$ 2nd test: second puncture at the ear, approximately $1 \mathrm{~cm}$ lateral from the first one at the left or right ear.

${ }^{4}$ All 3 minimally invasive sampling locations at the ears.

${ }^{5}$ Based on receiver operating characteristics (ROC) analyses. 
comparison of the AUROC curves from the 3 different capillary sampling locations showed no significant differences.

The average coefficients of variation for analyzing BHB concentrations with the FSP-Neo were $2.6 \%$ (inter-assay) and $11.7 \%$ (intra-assay), respectively. The coefficient of variation for the reference test used at the CCPU was $1.32 \%$ for analyzing the BHB concentrations.

\section{DISCUSSION}

For the interpretation of the results from capillary blood obtained by the FSP-Neo, it is important to evaluate this device by comparing it with a reference standard. Comparing the results of the FSP-Neo with other evaluated handheld meters, the results were comparable with most devices: Mahrt et al. (2014) reported a $\rho_{\mathrm{s}}$ for the NovaVet device of $\rho_{\mathrm{s}}=0.87$ with a mean \pm standard deviation BHB difference of -0.07 $\pm 0.42 \mathrm{mmol} / \mathrm{L}$ compared with the laboratory results. The optimized threshold for the NovaVet device was $1.2 \mathrm{mmol} / \mathrm{L}$ with Se and Sp of 97 and $82 \%$, respectively. Iwersen et al. (2013) evaluated 2 different handheld devices and reported a $\rho_{\mathrm{s}}=0.94$ with a mean \pm standard deviation BHB difference of $0.04 \pm 0.15 \mathrm{mmol} / \mathrm{L}$ for the FreeStyle Precision device and $\rho_{\mathrm{s}}=0.80$ with a mean \pm standard deviation BHB difference of -0.12 $\pm 0.22 \mathrm{mmol} / \mathrm{L}$ for the GlucoMen LX Plus handheld meter. For the FreeStyle Precision at an optimized threshold of $1.2 \mathrm{mmol} / \mathrm{L}$, a similar Se and Sp of $98 \%$ and $100 \%$, as found in the present study for the FSPNeo device, was reported. For the GlucoMen LX Plus, an equal optimized threshold as with the FSP-Neo was reported but a lower Se and Sp with $80 \%$ and $86 \%$ were determined. Considering the results of these and our studies, consistency for BHB determination with different electronic handheld meters in different farms with varying environmental conditions can be assumed.

The BHB concentration in capillary blood analyzed with the FSP-Neo showed a significant difference compared with the reference test. For practical use, an optimal classification into groups of SCK or healthy cows is more important; therefore, adjustments for the threshold were made. To identify SCK in capillary blood obtained from an ear with the FSP-Neo device, an optimized threshold of $1.3 \mathrm{mmol} / \mathrm{L}$ should be used. With this threshold, the results from ROC analyses for capillary blood were excellent; overall accuracies were AUROC $\geq 90 \%$ with a Se of $100 \%$ for all 3 sampling locations and a Sp of at least 93\%. Kanz et al. (2015) evaluated the use of different handheld meters for BHB testing in capillary blood obtained from the skin of the exterior vulva. For the FreeStyle Precision and the
NovaVet device, similar $\rho_{\mathrm{s}}=83 \%$ and $73 \%$ with lower mean \pm standard deviation BHB differences of $0.08 \pm$ $0.19 \mathrm{mmol} / \mathrm{L}$ and $-0.01 \pm 0.43 \mathrm{mmol} / \mathrm{L}$, respectively, were described. In that study, an optimized threshold for the FreeStyle Precision of $1.0 \mathrm{mmol} / \mathrm{L}$ for detecting SCK, with corresponding Se of $100 \%$ and Sp of $76 \%$, was reported. For the NovaVet device, the optimized threshold was $1.1 \mathrm{mmol} / \mathrm{L}$ with corresponding Se of $89 \%$ and Sp of $84 \%$.

The reason for the greater variation for BHB concentrations measured with the FSP-Neo device in capillary blood than in coccygeal blood compared with the reference test remains speculative. Compared with the reference test, the Bland and Altman plots showed that the measured BHB concentrations were systematically higher in capillary blood. Therefore, optimized thresholds for identifying SCK in capillary blood were determined. The ROC analyses showed that, when these optimized thresholds were applied, the FSP-Neo device is suitable to detect SCK in capillary blood of dairy cows.

The Bland and Altman plots showed only slight differences between the 3 capillary sampling locations at the ears and the results of the mixed model analyses confirmed that the sampling site had no influence on the measured BHB concentration. Hence, we conclude that all 3 sampling locations at the ear are suitable for ketone testing.

Another objective of this study was to evaluate the influence of squeezing the skin to get an adequate amount of capillary blood for the measurement on the determined BHB concentration. The comparison of the mean difference in BHB concentrations in both groups (no squeezing/squeezing) with the reference each showed a statistical significant difference of $0.06 \mathrm{mmol} / \mathrm{L}$. From a clinical perspective, however, this effect of squeezing is negligible for BHB measurements in capillary ear blood using the handheld device. Because the analyzed specimen (i.e., coccygeal vs. capillary blood) showed a greater effect on the BHB concentration, sampling site specific threshold should be used for diagnosing SCK.

Inter- and intra-assay coefficients of variations were calculated to evaluate the reproducibility and repeatability of the test results. The previously reported average inter- and intra-assay coefficients of variations for another handheld meter, the FreeStyle Precision device, were 7.2 and $7.9 \%$ for capillary blood from the skin of the exterior vulva (Kanz et al., 2015), and 5.8 and $5.7 \%$ for whole blood from a tail vessel (Iwersen et al., 2013). The measurements from capillary blood at the ear yielded similar average inter- and intra-assay coefficients of variation of 2.6 and $11.7 \%$, respectively. Compared with studies using automated wet chemistry analyzers for measuring BHB concentrations (Moallem 
et al., 2012; Sepúlveda-Varas et al., 2015), the coefficients of variation found in this study were greater for the electronic handheld device. As clinical laboratory instruments are used for precise measurements within a sample, field tests are expected to show greater variations (Vap and Weiser, 2007), but deemed as beneficial as they provide the test results on farm within a few minutes. Additionally, because the coefficients of variation were below $15 \%$ as requested by the European Medicines Agency (EMEA, 2011), the results can be considered as acceptable.

\section{CONCLUSIONS}

Despite the limitations of the study, for instance the low prevalence of SCK on the farm, the detection of HYK in capillary blood from an ear with the FSP-Neo is an additional option for monitoring SCK under field conditions. Particularly, the excellent test characteristics after the ROC-based adjustments of the thresholds allow the discrimination between healthy animals and cows suffering from HYK. Based on the results of our study, additional research including farms with a greater prevalence of SCK, farms without preventing strategies for HYK, and without constant monitoring for HYK is needed to test the external validity of the test results. Because capillary blood is easily achievable from the ear, particularly if animals are fixed in headlocks for routine checkups, this technique is a promising tool for the identification of dairy cows suffering from HYK.

\section{ACKNOWLEDGMENTS}

We thank the staff from FirstFarms Slovakia as well as Sonja Siekiera, Patrick Schmidseder, and Peter Kanz (all from the University of Veterinary Medicine Vienna, Vienna, Austria) for their technical assistance. The author(s) received no financial support for the research, authorship, or publication of this article.

\section{REFERENCES}

Berge, A. C., and G. Vertenten. 2014. A field study to determine the prevalence, dairy herd management systems, and fresh cow clini- cal conditions associated with ketosis in western European dairy herds. J. Dairy Sci. 97:2145-2154.

Bland, J. M., and D. Altman. 1986. Statistical methods for assessing agreement between two methods of clinical measurement. Lancet $1: 307-310$.

Bland, J. M., and D. G. Altman. 1995. Multiple significance tests: The Bonferroni method. BMJ 310:170.

Duffield, T. F., D. Sandals, K. E. Leslie, K. Lissemore, B. W. McBride, J. H. Lumsden, P. Dick, and R. Bagg. 1998. Efficacy of monensin for the prevention of subclinical ketosis in lactating dairy cows. J. Dairy Sci. 81:2866-2873.

EMEA (European Medicines Agency). 2011. Guideline on bioanalytical method validation. Accessed May 3, 2015. http://www.ema.europa. eu/docs/en_GB/document_library/Scientific_guideline/2011/08/ WC500109686.pdf.

Fawcett, T. 2006. An introduction to ROC analysis. Pattern Recognit. Lett. 27:861-874.

Iwersen, M., D. Klein-Jöbstl, M. Pichler, L. Roland, B. Fidlschuster, I. Schwendenwein, and M. Drillich. 2013. Comparison of 2 electronic cowside tests to detect subclinical ketosis in dairy cows and the influence of the temperature and type of blood sample on the test results. J. Dairy Sci. 96:7719-7730.

Kanz, P., M. Drillich, D. Klein-Jöbstl, B. Mair, S. Borchardt, L. Meyer, I. Schwendenwein, and M. Iwersen. 2015. Suitability of capillary blood obtained by a minimally invasive lancet technique to detect subclinical ketosis in dairy cows by using 3 different electronic handheld devices. J. Dairy Sci. 98:6108-6118.

Mahrt, A., O. Burfeind, and W. Heuwieser. 2015. Evaluation of hyperketonemia risk period and screening protocols for early-lactation dairy cows. J. Dairy Sci. 98:3110-3119.

Mahrt, A., O. Burfeind, R. Voigtsberger, A. Müller, and W. Heuwieser. 2014. Evaluation of a new electronic handheld meter for measurement of $\beta$-hydroxybutyric acid in dairy cows. Tierarztl. Prax. Ausg. G Grosstiere Nutztiere 42:5-10.

Moallem, U., A. Rozov, E. Gootwine, and H. Honig. 2012. Plasma concentrations of key metabolites and insulin in late-pregnant ewes carrying 1 to 5 fetuses. J. Anim. Sci. 90:318-324.

Oetzel, G. R. 2004. Monitoring and testing dairy herds for metabolic disease. Vet. Clin. North Am. Food Anim. Pract. 20:651-674.

Pichler, M., A. Damberger, T. Arnholdt, I. Schwendenwein, J. Gasteiner, M. Drillich, and M. Iwersen. 2014. Evaluation of 2 electronic handheld devices for diagnosis of ketonemia and glycemia in dairy goats. J. Dairy Sci. 97:7538-7546.

Pineda, A., and F. C. Cardoso. 2015. Technical note: Validation of a handheld meter for measuring $\beta$-hydroxybutyrate concentrations in plasma and serum from dairy cows. J. Dairy Sci. 98:8818-8824.

Sepúlveda-Varas, P., D. M. Weary, M. Noro, and M. A. von Keyserlingk. 2015. Transition diseases in grazing dairy cows are related to serum cholesterol and other analytes. PLoS ONE 10:e0122317.

Suthar, V. S., J. Canelas-Raposo, A. Deniz, and W. Heuwieser. 2013 Prevalence of subclinical ketosis and relationships with postpartum diseases in European dairy cows. J. Dairy Sci. 96:2925-2938.

Swets, J. A. 1988. Measuring the accuracy of diagnostic systems. Science 240:1285-1293.

Vap, L. M., and M. G. Weiser. 2007. Field chemistry analysis. Vet. Clin. North Am. Food Anim. Pract. 23:427-442.

Youden, W. J. 1950. Index for rating diagnostic tests. Cancer 3:32-35. 